\title{
Postoperative Changes in Fecal Bacterial Communities and Fermentation Products in Obese Patients Undergoing Bilio-Intestinal Bypass
}

\begin{abstract}
Vania Patrone ${ }^{1 *}$, Elia Vajana ${ }^{2}$, Andrea Minuti ${ }^{2}$, Maria L. Callegari ${ }^{1}$, Alessandro Federico ${ }^{3}$, Carmela Loguercio ${ }^{3}$, Marcello Dallio ${ }^{3}$, Salvatore Tolone ${ }^{4}$, Ludovico Docimo ${ }^{4}$ and Lorenzo Morelli ${ }^{1}$

${ }^{1}$ Instituto di Microbiologia, Facoltà di Scienze Agrarie, Alimentari e Ambientali, Università Cattolica del Sacro Cuore, Piacenza, Italy, ${ }^{2}$ Istituto di Zootecnica, Facoltà di Scienze Agrarie, Alimentari e Ambientali, Università Cattolica del Sacro Cuore, Piacenza, Italy, ${ }^{3}$ Division of Hepatogastroenterology, Second University of Naples, Naples, Italy, ${ }^{4}$ Division of General and Bariatric Surgery, Second University of Naples, Naples, Italy
\end{abstract}

OPEN ACCESS

Edited by:

Zhongtang Yu,

The Ohio State University, USA

Reviewed by:

Jeffrey David Galley,

Baylor College of Medicine, USA

Seungha Kang,

Commonwealth Scientific and Industrial Research Organisation

(CSIRO), Australia

${ }^{*}$ Correspondence:

Vania Patrone

vania.patrone@unicatt.it

Specialty section:

This article was submitted to

Microbial Symbioses,

a section of the journal

Frontiers in Microbiology

Received: 14 December 2015 Accepted: 05 February 2016

Published: 23 February 2016

Citation:

Patrone V, Vajana E, Minuti A

Callegari ML, Federico $A$,

Loguercio C, Dallio M, Tolone S,

Docimo L and Morelli L (2016)

Postoperative Changes in Fecal

Bacterial Communities and

Fermentation Products in Obese

Patients Undergoing Bilio-Intestinal

Bypass. Front. Microbiol. 7:200

doi: 10.3389/fmich.2016.00200
We assessed the gut microbial ecology of 11 severely obese patients before and after bilio-intestinal bypass (BIB). Fecal samples were evaluated for microbial communities using $16 \mathrm{~S}$ rDNA lllumina sequencing, real-time PCR targeting functional genes, and gas chromatography of short chain fatty acids (SCFAs). At 6 months after surgery, subjects exhibited significant improvements in metabolic markers (body weight, glucose, and lipid metabolism) compared with baseline. The fecal microbiota of post-surgery individuals was characterized by an overall decrease of bacterial diversity, with a significant reduction in Lachnospiraceae, Clostridiaceae, Ruminococcaceae, Eubacteriaceae, and Coriobacteriaceae. On the contrary, there were significant increases of genera Lactobacillus, Megasphaera, and Acidaminococcus and the family Enterobacteriaceae. The $\mathrm{pH}$ was decreased in fecal samples from patients after BIB and SCFA profiles were altered, with lower percentages of acetate and propionate and higher levels of valerate and hexanoate. Some changes in the bacterial populations were associated with variations in the patients' metabolic health parameters, namely Gemmiger and glucose, Lactobacillus and glucose, and Faecalibacterium and triglycerides. The results from this study of BIB patients furthers our understanding of the composition of gut microbiota and the functional changes that may be involved in improving obesity-related conditions following weight-loss surgery.

Keywords: obesity, gut microbiota, weight loss, bariatric surgery, short chain fatty acids, illumina sequencing, $16 \mathrm{~S}$ rRNA

\section{INTRODUCTION}

Emerging evidence suggests that microbes that reside in the human gastrointestinal tract may be involved in the development of obesity. The gut microbiota contributes to weight gain by promoting storage of calories from the diet as fat (Bäckhed et al., 2004; Turnbaugh et al., 2006). Several studies in humans and animal models have highlighted differences in the composition of 
the gut microbiota between obese and lean subjects, sometimes yielding contrasting results (Ley et al., 2005, 2006; Turnbaugh et al., 2006, 2008, 2009; Schwiertz et al., 2010).

In addition, the association between gut microbiota and obesity is supported by studies exploring the effects of bariatric surgery on gut microbial community composition. This surgery is the most effective current option for treating severe obesity. By reducing intake or absorption of calories, surgical intervention produces rapid and long-lasting weight loss and leads to an improvement of many obesity-related conditions including type-2 diabetes, hypertension, and high cholesterol. Zhang et al. (2009) showed that individuals subjected to Roux-en-Y gastric bypass (RYGB), the most commonly performed bariatric procedure, have higher numbers of Enterobacteriaceae bacteria (phylum Proteobacteria) and lower proportions of Clostridium bacteria (phylum Firmicutes) and methanogens compared with obese individuals. A subsequent study by Furet et al. (2010) included obese subjects before, and 3 and 6 months after RYGB, along with lean controls. Their results revealed that the prevalence of the Bacteroides/Prevotella group was lower in obese individuals compared with lean subjects, but it increased after gastric surgery. On the contrary, populations of Bifidobacterium and Lactobacillus/Leuconostoc/Pediococcus decreased after surgery. At the phylum level, obese patients had a high Firmicutes to Bacteroidetes ratio before RYGB that decreased 3 and 6 months postoperatively.

Li et al. (2011) evaluated the effects of RYGB in a nonobese rat model, and found a striking increase of Proteobacteria as well as a decrease of both Firmicutes and Bacteroidetes compared with sham-operated rats. In a key study, Liou et al. (2013) performed 16S rDNA sequencing in three groups of mice, which underwent RYGB, sham surgery, or sham surgery coupled with caloric restriction. The RYGB produced specific changes in the gut microbial community, i.e., increases in Bacteroidetes, Verrucomicrobia, and Proteobacteria (Alistipes, Akkermansia, and Escherichia, respectively, at the genus level) compared with preoperative levels. Even more important, transferring gut microbiota from RYGB-mice to germ-free mice induced weight loss in those animals compared with mice receiving gut samples from sham-treated mice. These results suggest that changes in the population of microbial organisms in the gastrointestinal tract may mediate some of the beneficial effects of bariatric surgery on the obese host.

Although RYGB is the most widely used type of bariatric surgery in the world, there are several available surgical methods for the treatment of morbidly obese patients. Bilio-intestinal bypass (BIB) surgery (Eriksson, 1981) consists of disabling a large portion of the absorptive surfaces within the small bowel using a shunt between the beginning and end-part, and connecting the disabled small bowel sling to the gall-bladder to improve enterohepatic circulation of bile acids. It is unknown if the changes in microbial ecology observed for RYGB can be generalized to other types of bariatric surgery; therefore, we studied fecal samples using $16 \mathrm{~S}$ rDNA next generation sequencing to determine the impact of BIB on the gut microbial composition and environment, and pinpoint the association between the microbial rearrangement and variations in the host phenotype.

\section{MATERIALS AND METHODS}

\section{Patients}

Twenty severely obese patients were evaluated for bariatric surgery. Eleven subjects were eligible for BIB, by fulfilling the following criteria: (1) morbid obesity [body mass index $\left(\mathrm{BMI}\right.$; in $\left.\mathrm{kg} \mathrm{m}^{-2}\right)>40$ ] or severe obesity (BMI > 35), at least one comorbidity factor (arterial hypertension, diabetes mellitus) for $\geq 5$ years, and resistance to medical treatment; (2) absence of medical or psychological contraindications for bariatric surgery; (3) absence of current excessive drinking, as defined by an average daily consumption of alcohol of 20 $\mathrm{g} \mathrm{d}^{-1}$ for women and $30 \mathrm{~g} \mathrm{~d}^{-1}$ for men, and no history of excessive drinking for a period $>2$ years at any time in the past 20 years; (4) absence of long-term consumption of hepatotoxic drugs; and (5) negative screening for chronic liver diseases, including hepatitis B surface antigen and hepatitis $\mathrm{C}$ virus antibodies, and other known liver diseases such as Wilson's disease, hemochromatosis, $\alpha 1$-antitrypsin deficiency, or autoimmune hepatitis; and (6) no malignant diseases. Informed written consent was obtained from all patients, and the study was conducted in conformity with the Helsinki Declaration. The Ethics Committee of the University Hospital Second University of Naples, Naples (Italy) approved the study. At baseline, all patients were evaluated by a multidisciplinary team consisting of dieticians, gastroenterologists, psychiatrists, an anesthesiologist, a cardiologist, an endocrinologist, and surgeons. The main demographic characteristics of patients are reported in Table 1.

\section{Biochemical Evaluation}

Subjects fasted overnight for at least $12 \mathrm{~h}$ before an antecubital vein catheter was inserted for blood collection. The following clinical and biological features were assessed before surgery: BMI, alanine aminotransferases (ALT), gamma-glutamyl transpeptidase $(\gamma \mathrm{GT})$, serum triglycerides, cholesterolemia, fasting blood glucose, and fasting insulin. The energy intake from diet was obtained by recording individual dietary habits using the Winfood Software 2.0 package (Medimatica s. r. 1., Martinsicuro, Italy) program, which calculates calories on the basis of the quantities and qualities of consumed food (Musso et al., 2005). Biochemical characteristics of patients at baseline are reported in Table 1. Parameters related to weight loss, glucose, and lipid metabolism were also evaluated 6 months after bariatric surgery.

\section{DNA Extraction}

Fecal samples were collected before and 6 months after BIB. Fifty milli gram of feces were homogenized in $500 \mu \mathrm{l}$ of $10 \mathrm{mg} / \mathrm{ml}$ lysozyme solution in Tris-sucrose buffer $(50 \mathrm{mM}$ Tris- $\mathrm{HCl}, \quad 40 \mathrm{mM}$ EDTA, $0.75 \mathrm{M}$ sucrose, $\mathrm{pH}$ 8.0) and incubated for $1 \mathrm{~h}$ at $37^{\circ} \mathrm{C}$. The DNA was purified using the Maxwell ${ }^{\circledR} 16$ DNA Purification Kit and the Maxwell ${ }^{\circledR} 16$ Instrument (Promega, Madison, WI, USA), according to the manufacturer's instructions. The DNA quality was checked by 
TABLE 1 | Baseline demographic and biochemical characteristics of the patients studied.

\begin{tabular}{|c|c|c|c|c|c|c|c|c|c|c|c|}
\hline & \multicolumn{11}{|c|}{ Patients } \\
\hline & 1 & 2 & 3 & 4 & 5 & 6 & 7 & 8 & 9 & 10 & 11 \\
\hline Age (years) & 45 & 56 & 46 & 50 & 48 & 56 & 64 & 58 & 35 & 58 & 41 \\
\hline $\operatorname{Sex}(F / M)$ & $\mathrm{F}$ & $\mathrm{F}$ & $\mathrm{F}$ & $M$ & $\mathrm{~F}$ & $\mathrm{M}$ & $\mathrm{F}$ & $\mathrm{F}$ & $\mathrm{F}$ & $\mathrm{F}$ & $\mathrm{F}$ \\
\hline Body weight (kg) & 102.40 & 80.60 & 125.00 & 107.00 & 73.40 & 113.00 & 115.00 & 101.90 & 110.00 & 103.00 & 145.00 \\
\hline $\mathrm{BMl}\left(\mathrm{kg} \mathrm{m}^{-2}\right)$ & 45.90 & 42.49 & 51.42 & 39.21 & 33.37 & 44.08 & 53.91 & 46.65 & 53.80 & 52.41 & 58.82 \\
\hline Diabetes mellitus (Y/N) & Y & $\mathrm{N}$ & Y & Y & Y & $\mathrm{N}$ & Y & $N$ & $\mathrm{~N}$ & Y & $\mathrm{N}$ \\
\hline Arterial hypertension $(\mathrm{Y} / \mathrm{N})$ & Y & Y & Y & $N$ & Y & Y & $\mathrm{N}$ & $\mathrm{N}$ & Y & Y & $\mathrm{N}$ \\
\hline Insulinemia (mIU L ${ }^{-1}$ ) & 16.00 & 17.00 & 11.80 & 24.00 & 16.00 & 31.60 & 11.80 & 15.00 & 18.00 & 21.00 & 21.90 \\
\hline Glycemia (mg dL ${ }^{-1}$ ) & 117.00 & 83.00 & 129.00 & 109.00 & 130.00 & 99.00 & 129.00 & 98.00 & 92.00 & 107.00 & 99.00 \\
\hline $\operatorname{ALT}\left(I \cup L^{-1}\right)$ & 48.00 & 61.00 & 46.00 & 85.00 & 54.00 & 56.00 & 40.00 & 78.00 & 26.00 & 89.00 & 64.00 \\
\hline yGT (IU L $\left.L^{-1}\right)$ & 38.00 & 66.00 & 64.00 & 54.00 & 49.00 & 94.00 & 84.00 & 42.00 & 51.00 & 43.00 & 89.00 \\
\hline Total cholesterol (mg dL ${ }^{-1}$ ) & 179.00 & 254.00 & 163.00 & 154.00 & 179.00 & 265.00 & 163.00 & 216.00 & 190.00 & 237.00 & 214.00 \\
\hline Triglycerides (mg dL ${ }^{-1}$ ) & 75.00 & 188.00 & 108.00 & 128.00 & 202.00 & 148.00 & 108.00 & 101.00 & 92.00 & 93.00 & 141.00 \\
\hline Caloric intake $\left(\mathrm{kcal} \mathrm{d}^{-1}\right)$ & 3825.50 & 2505.40 & 3297.79 & 3511.70 & 3379.00 & 3809.10 & 1949.20 & 3468.21 & 1410.10 & 3446.90 & 2486.30 \\
\hline
\end{tabular}

F, female; M, male; Y, yes; N, no; BMI, body mass index; ALT, alanine aminotransferases; $\gamma$ GT, gamma-glutamyl transpeptidase; IU, international units.

agarose gel electrophoresis and the DNA concentration was determined using the Qubit HS dsDNA fluorescence assay (Life Technologies, Carlsbad, CA, USA).

\section{Illumina Sequencing of the 16S rRNA Gene}

The V4 region of the bacterial 16S rRNA gene was PCR amplified using the primers 520F and $802 \mathrm{R}$, which have a high overall coverage and reasonably good domain specificity (Klindworth et al., 2013). To allow multiplexing during Illumina sequencing, both forward and reverse primers were modified by the addition of a seven base long tag. For each sample the DNA amplification was performed in triplicate using $5 \mathrm{ng}$ of DNA for each reaction. The PCR conditions were previously reported (Claesson et al., 2009). The resulting amplicons were checked by capillary electrophoresis using a QIAxcel (Qiagen $\mathrm{GmbH}$, Hilden, Germany). All amplicons were purified using the MinElute PCR purification kit (Qiagen $\mathrm{GmbH}$, Hilden, Germany) and then mixed together in equimolar concentrations. The library was sequenced on a MiSeq System using the MiSeq Reagent Kit v3 at a $2 \times 250$ bp read length configuration Paired-end sequencing $(2 \times 250 \mathrm{bp})$ was carried out on an Illumina MiSeq sequencer (Fasteris, Genève, Switzerland).The OBITools programs (www.prabi.grenoble.fr/trac/OBITools) were used to analyze the DNA sequence reads from Illumina, including assembling pair-end reads, assigning each read to its original sample, and de-replicating reads into unique sequences. To denoise the sequence dataset, singletons, and short sequences ( $<20$ nucleotides) were first removed using the obigrep command and then the sequences were cleaned of PCR/sequencing errors and chimeras using the obiclean program. For taxonomic assignment of sequences, a reference database was built using the ecoPCR program, which performs an in silico PCR to extract all sequences from the EMBL nucleotide library that may be amplified by the 520F/802R primer pair. The taxons were assigned using the EcoTag program. To further eliminate putative PCR and/or sequencer artifacts, bioinformatic
TABLE 2 | Real-time PCR primers used to amplify microbial functional genes.

\begin{tabular}{llll}
\hline Primers & $\begin{array}{l}\text { Target } \\
\text { Gene }\end{array}$ & Runctional Group & \\
\hline ACS_f/ACS_r & acsB & Acetogens & Gagen et al., 2010 \\
qmcrA-F/qmcrA-R & $m c r A$ & Methanogens & Denman et al., 2007 \\
DRS1F+/DSR-R & $d s r A$ & Sulfate-reducing bacteria & Kondo et al., 2004 \\
BcoAf/BcoAr & BCOAT & Butyrate-producing bacteria & Louis and Flint, 2007
\end{tabular}

acsB, acetyl-CoA synthase gene; mcrA, methyl-coenzyme $M$ reductase gene; dsrA, dissimilatory (bi)sulfite reductase gene; BCOAT, butyryl-CoA: acetate CoA transferase gene.

filtering was used and only sequences with a $90 \%$ identity to the sequences present in GenBank were kept for further analysis.

\section{Real-Time PCR}

To determine the presence of hydrogen consumers and butyrate producers, PCR was performed on fecal DNA using the primer sets listed in Table 2. The qPCR assays were run using the StepOne ${ }^{\mathrm{TM}}$ Plus Real-Time PCR System (Applied Biosystems, Foster City, CA, USA). The amplification reaction was carried out in $20 \mu \mathrm{l}$ containing $2 \mu \mathrm{l}$ DNA, $0.4 \mathrm{mM}$ of each forward and reverse primer, and $10 \mu \mathrm{l}$ of KAPA SYBR FAST qPCR Master Mix (Kapa Biosystems, Wilmington, MA, USA). Standard curves were determined using plasmids containing amplified fragments of acs, $d s r A, m c r A$, or genomic DNA from reference strains for $B c o A T$. Results were expressed as gene copy numbers per gram wet feces.

\section{Metabolite Analysis}

Fecal samples (1.0 g weight) were diluted at a ratio $1: 4(\mathrm{w} / \mathrm{v})$ in sterile distilled water. The $\mathrm{pH}$ of the fecal extracts was measured using a $\mathrm{pH}$ meter (GLP 21; Crison Instruments SA, Alella, Barcelona, Spain). The samples were then vortexed for 
$1 \mathrm{~min}$ and the homogenate centrifuged at $10,000 \times \mathrm{g}$ for $15 \mathrm{~min}$ at $10^{\circ} \mathrm{C}$. The supernatant was transferred to a new tube and stored at $-20^{\circ} \mathrm{C}$ until analysis. The Megazyme L-and D-Lactate Assay Kit (Megazyme International Ltd., Wicklow, Ireland) was used to detect D- and L-lactate. Urea was analyzed at $37^{\circ} \mathrm{C}$ via a clinical auto-analyzer (ILAB 600, Instrumentation Laboratory, Lexington, MA, USA) using a kit purchased from Instrumentation Laboratory (IL Test). An aliquot of fecal extract was acidified with $0.12 \mathrm{M}$ oxalic acid and frozen at $-20^{\circ} \mathrm{C}$ until the analysis of SCFAs. The SCFA concentration in fecal extract was analyzed by gas chromatography (model 7820A; Agilent Technologies, Santa Clara, CA, USA) using a capillary column $(30 \mathrm{~m} \times 250 \mu \mathrm{m} \times 0.25 \mu \mathrm{m}$; DB-FFAP, Agilent J\&W GC column) and a flame-ionization detector. The oven temperature was held at $60^{\circ} \mathrm{C}$ for $5 \mathrm{~min}$ and then increased by $5^{\circ} \mathrm{C} / \mathrm{min}$ to $170^{\circ} \mathrm{C}$. The injector temperature was $250^{\circ} \mathrm{C}$ and the detector temperature was $300^{\circ} \mathrm{C}$. The injector was equipped with a liner of glass wool to separate particles of dirt from the sample. The samples were injected by auto-sampler $(1 \mu \mathrm{L}$ volume $)$ using the split method and a 25:1 splitting ratio. Hydrogen and air were used for flame ionization detection. The carrier gas was nitrogen, with a constant flow of $1.78 \mathrm{~mL} / \mathrm{min}$. Pivalic acid was used as an internal standard.

\section{Statistical Analysis}

To verify an adequate sequencing depth of the fecal microbiota, we performed rarefaction analysis on raw counts using an Rbased protocol (http://www.jennajacobs.org/R/rarefaction.html). Alpha and beta diversity analysis was performed in $\mathrm{R}$ (version 3.1.2) (R Core Team, 2014) using the Vegan package (Oksanen et al., 2014). To avoid bias due to different sampling depths, we first rarefied the OTU abundance table by randomly subsampling each sample to the smallest data size. Differences between fecal bacterial communities were visualized using non-metric multidimensional scaling (NMDS) on the basis of the Bray-Curtis distance matrix. Hierarchical clustering using Spearman rank correlation distance and Ward linkage was performed on relative abundance values at the genus level using the hclust function of Vegan. To identify bacterial taxa that were differentially abundant in the gut microbiota of obese patients before and after surgery, we utilized the LEfSe algorithm (Segata et al., 2011) implemented in the Galaxy module (http://huttenhower. sph.harvard.edu/galaxy/). We used per-sample normalization on bacterial relative abundance data. The default value of alpha was 0.05 and the logarithmic LDA score threshold was 2 . A principal component analysis (PCA) was carried out using the prcomp function in $\mathrm{R}$ to explore relationships between bacterial populations that significantly changed after BIB and clinical parameters of the host. To investigate associations among bacterial populations and SCFA concentrations, linear mixed-effects models were fitted by maximum likelihood (ML) with the lme function in the nlme $\mathrm{R}$ package (Pinheiro et al., 2013). After standardization to zero mean and unit variance, bacteria populations were tested as fixed independent effects, and SCFA concentrations as response variables. To take into account the repeated-measures design of the experiment, subjects were modeled as random factors. Likewise, associations between bacterial groups that significantly changed after BIB and anthropometric and biochemical measurements, including dietary caloric intake, were also assessed. The Bonferroni correction was used as an adjustment method for multiple testing, with a chosen significance level of $P \leq 0.05$ for multiple comparisons of the associations between bacterial genera and SCFAs, and of $P \leq 0.20$ for multiple comparisons between bacterial genera and bio-clinical markers. To compare population means for statistical significance, the Shapiro-Wilks test was used to check for normality, and paired $t$-test or Wilcoxon rank-sum tests were performed using either R or GraphPad Prism version 5 (Graphpad Software, San Diego, CA, USA).

\section{Nucleotide Sequence Accession}

The $16 \mathrm{~S}$ sequence data are publicly available on Dryad repository (http://dx.doi.org/10.5061/dryad.j82n2).

\section{RESULTS}

\section{Bio-Clinical Characteristics of Surgical Patients}

As expected, obese individuals showed a significant reduction in BMI and body weight 6 months after surgical intervention (Table 3). Circulating glucose and insulin were also decreased, which indicated an amelioration of glucose homeostasis, as well as postoperative triglyceride and total cholesterol concentrations. An analysis of total food intake showed a drastic caloric reduction 6 months after surgery compared with the preoperative nutritional status (Table 3).

\section{Sequencing Coverage and Estimation of Fecal Bacterial Diversity}

In this study, the bacterial composition of the fecal samples was examined using an Illumina high-throughput sequencing technique. We generated a dataset consisting of 1,232,275 filtered high-quality and classifiable $16 \mathrm{~S}$ rRNA gene sequences, and the average number of sequences obtained for each individual was 56,013 (range: from 25,224 to 102,372). All sequences were clustered with representative sequences, and a $97 \%$ sequence identity cut-off was used. The total number of operational

TABLE 3 | Characteristics of the study population pre- (baseline) and post-surgery ( 6 months after bilio-intestinal bypass).

\begin{tabular}{|c|c|c|}
\hline Parameters & Pre-Surgery $(n=11)$ & Post-Surgery $(n=11)$ \\
\hline Body weight, kg & $106.94 \pm 19.41$ & $89.21 \pm 17.90^{\star \star *}$ \\
\hline BMI, $\mathrm{kg} \mathrm{m}^{-2}$ & $47.46 \pm 7.46$ & $40.68 \pm 5.88^{\star \star *}$ \\
\hline Insulin, mIU L $L^{-1}$ & $18.55 \pm 5.80$ & $13.55 \pm 2.16^{\star \star}$ \\
\hline Glucose, $\mathrm{mg} \mathrm{dL}^{-1}$ & $108.36 \pm 16.10$ & $93.36 \pm 10.93^{\star * *}$ \\
\hline Cholesterol, mg dL ${ }^{-1}$ & $201.27 \pm 38.45$ & $152.09 \pm 33.27^{\star \star *}$ \\
\hline Triglycerides, $\mathrm{mg} \mathrm{dL}^{-1}$ & $125.82 \pm 40.56$ & $102.00 \pm 35.32^{\star *}$ \\
\hline Caloric intake, kcal d $\mathrm{d}^{-1}$ & $3008.11 \pm 799.31$ & $1540.28 \pm 378.16^{\star \star \star}$ \\
\hline
\end{tabular}

$B M I$, body mass index. Values are the mean $\pm S D(n=11)$. Paired Student's $t$-test was performed to compare values before and after bilio-intestinal bypass. Asterisks indicate statistical significance $\left({ }^{\star \star} P<0.01 ;{ }^{\star \star \star} P<0.001\right)$. 

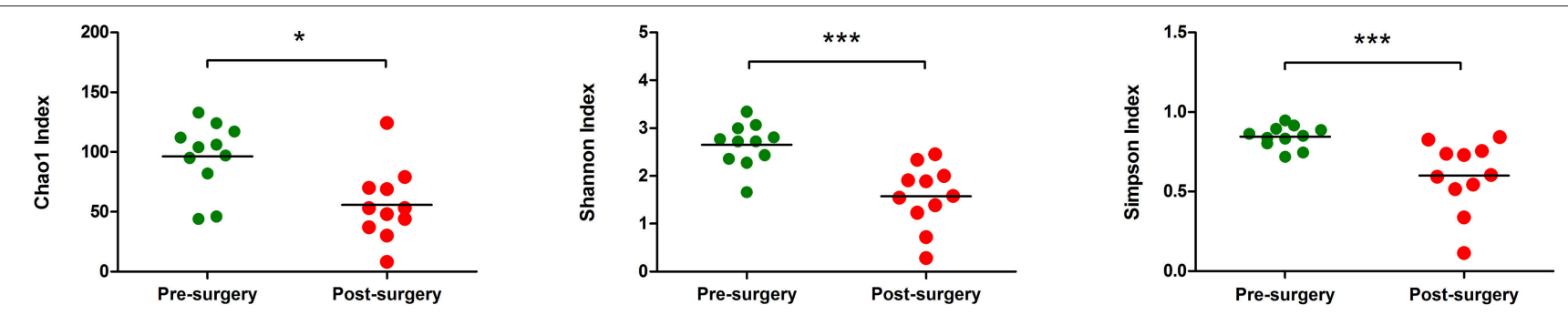

FIGURE 1 | Alpha diversity metrics (Chao 1, Shannon and Simpson indices) of OTU-level fecal bacterial communities from obese individuals; each point represents a sample. Paired Student's $t$-test was performed to compare alpha diversity estimates before and after bilio-intestinal bypass. Asterisks indicate statistical significance $\left({ }^{\star} P<0.05 ;{ }^{* \star *} P<0.001\right)$.

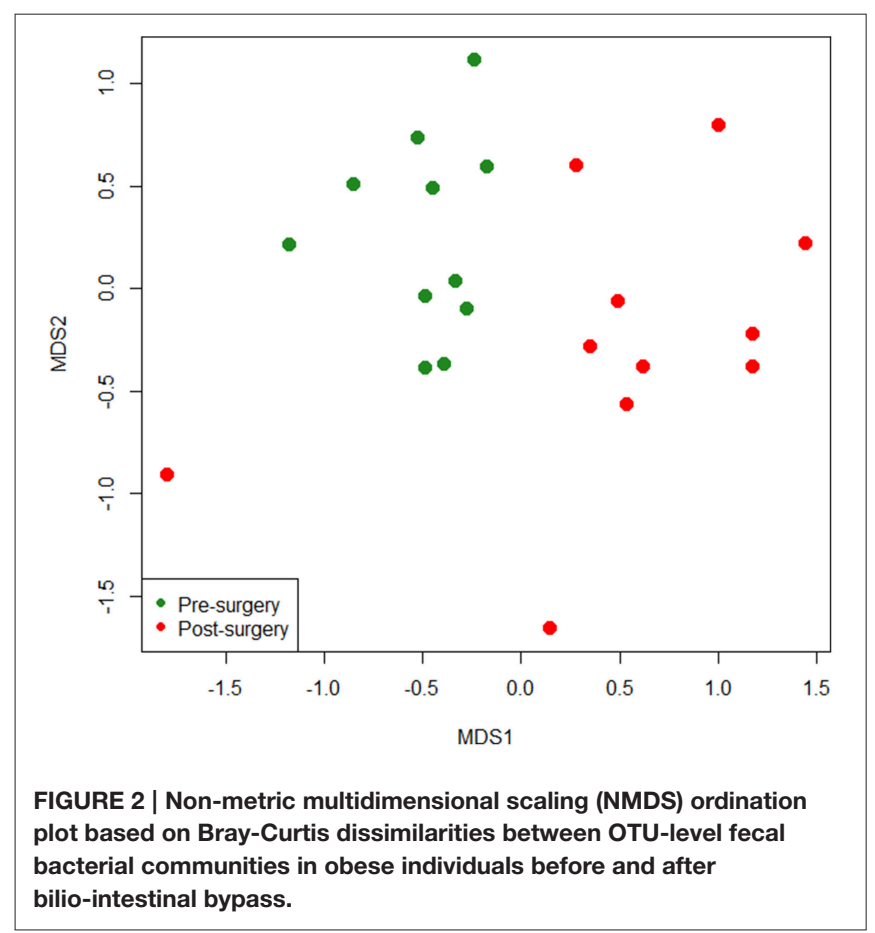

taxonomic units (OTUs) obtained was 1244. Rarefaction curves showed that, for each subject, bacterial species richness leveled off as sampling depth increased, indicating that the sequencing effort was sufficient and that the total diversity within the sample was captured (Supplementary Figure 1). They also suggested that the alpha diversity in pre-surgery samples was higher than in postsurgery samples. To confirm this observation, Chaol indexes were calculated and evaluated by paired $t$-test (Figure 1). There was a significant decrease in the mean microbial richness in post-surgery subjects compared with pre-surgery $(P=0.018)$. A diversity analysis based on Simpson and Shannon indexes revealed that the diversity of the intestinal microbiota at the OTU level was significantly higher in the fecal samples of presurgery subjects compared with post-surgery $(P=0.0009$ and $P=0.0008$, respectively). To compare the composition of the intestinal microbiota of patients before and after surgery, non-metric multi-dimensional scaling (NMDS) of a Bray-Curtis distance matrix based on the abundance of OTUs was employed. The NMDS revealed differences in the microbiota composition based on surgery (Figure 2). An apparent clustering pattern was identified for the participants before and after BIB. Points representing the intestinal microbiota composition of obese subjects and treated subjects clustered at the top left and the top right, respectively.

\section{Taxonomic Composition of Fecal Bacterial Communities}

Firmicutes, Actinobacteria, Bacteroidetes, Proteobacteria, and Verrucomicrobia constituted the five dominant bacterial phyla in the obese subjects under study, making up an average of 75.59, $21.62,0.89,0.82$, and $1.05 \%$ of the total sequences, respectively (Supplementary Figure 2). At the family level, the predominant taxa were Veillonellaceae, Bifidobacteriaceae, Ruminococcaceae, Lactobacillaceae, Lachnospiraceae, Erysipelotrichaceae, and Clostridiaceae (data not shown). A portion (4.4\%) of sequences belonging to the order Clostridiales was not assigned to any family. At the genus level, reads were assigned to 73 genera. A barplot representation of genus-level sequence data showed high inter-individual variability in the bacterial profile, indicating that relative abundances of each genus varied a lot between individuals, both before and after intervention (Figure 3B). The most abundant genus in the gut of obese individuals was Bifidobacterium, which accounted for $22.2 \%$ of the total number of sequences in both pre- and post-surgery samples. Lactobacillus was the second most abundant genus in postsurgery individuals whereas Roseburia was the second most abundant genus in pre-surgery samples. Megasphaera was detected only in post-surgery individuals where it represented $16.1 \%$ of the sequences. Hierarchical clustering of the microbiota community composition at the genus level showed that almost all post-surgery profiles clustered separately from their pre-surgery counterpart (Figure 3A).

\section{Supervised Comparison of the Gut Microbiota between Pre- and Post-surgery Patients}

Some of these microbial groups were present in different concentrations in the feces of pre- vs. post-surgery individuals; therefore, we attempted to highlight genera showing significant 


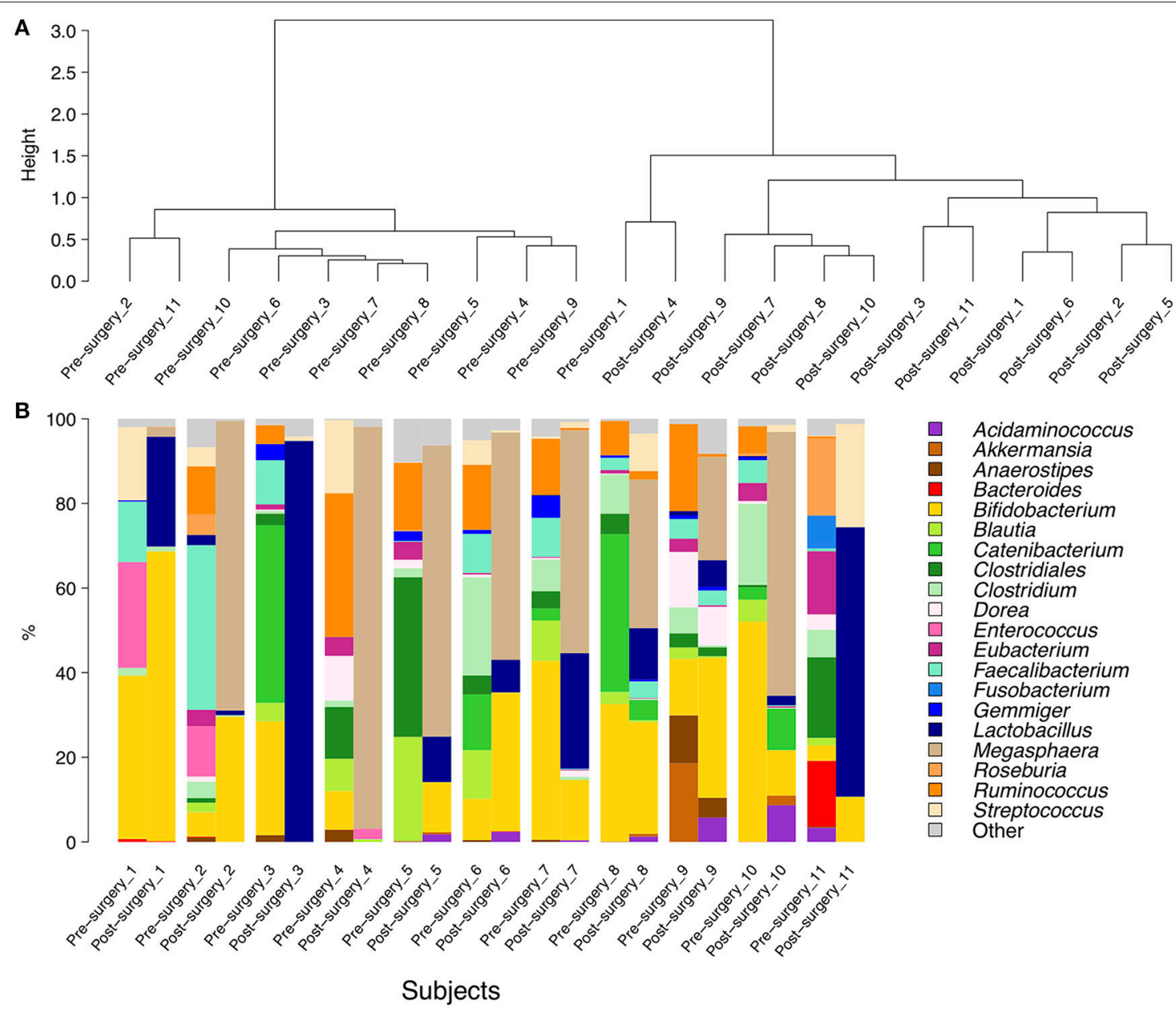

FIGURE 3 | Hierarchical clustering (A) and a barplot of relative abundances (B) of bacterial genera found within the fecal communities of obese patients. Only genera with a relative abundance $\geq 5 \%$ in at least one subject are represented. The dendrograms were calculated using Spearman's rank correlation and Ward-linkage clustering.

differences. The linear discriminant analysis effect size (LefSE) method identified a total of 31 bacterial groups that were differentially abundant in obese individuals before and after bariatric surgery (Figure 4 and Supplementary Figure 3). There were 19 groups of bacteria enriched in the fecal samples of pre-surgery individuals, namely, Clostridiales (the order and its families Lachnospiraceae, Ruminococcaceae, Clostridiaceae, and Eubacteriaceae); Coriobacteriales (the order and its family Coriobacteriaceae); Carnobacteriaceae (the family and its genus Granulicatella); and Lactococcus. Within Clostridiales, genera with a linear discriminant analysis (LDA) score $\geq 4$ were Faecalibacterium $(P=0.0019)$, Ruminococcus $(P=0.0007)$, Clostridium $(P=0.0001)$, Eubacterium $(P=0.0007)$, and Blautia $(P=0.0003)$. The bacterial groups enriched in post-surgery patients were Selenomonadales (the order, its family Veillonellaceae, and the two genera Megasphaera and Acidaminococcus), the family Enterobacteriaceae (from phylum Proteobacteria to order Enterobacteriales), and the genus Lactobacillus (from the class Bacilli to the family
Lactobacillaceae). Within these, genus Megasphaera had the highest LDA score of $5.33(P=0.0002)$, followed by Lactobacillus with a LDA of $4.93(P=0.0009)$ and Acidaminococcus with a LDA of $4.00(P=0.035)$.

\section{Associations between Key Bacterial Populations and Host Parameters}

Principal component analysis (PCA) (Supplementary Figure 4) indicated that some putative correlations could exist between significant bacterial populations and host clinical parameters. Univariate linear mixed models were then used to substantiate such relationships. Analysis revealed several significant associations but, after adjusting for multiple testing, only five models showed a $P \leq 0.20$. Among them, positive associations were found between Clostridium levels and blood insulin $(B$ $=0.728, P=0.019$; where $B$ is the estimated standardized regression coefficient and $P$ the related $P$-value after Bonferroni correction), Faecalibacterium and triglycerides $(B=0.610, P=$ 


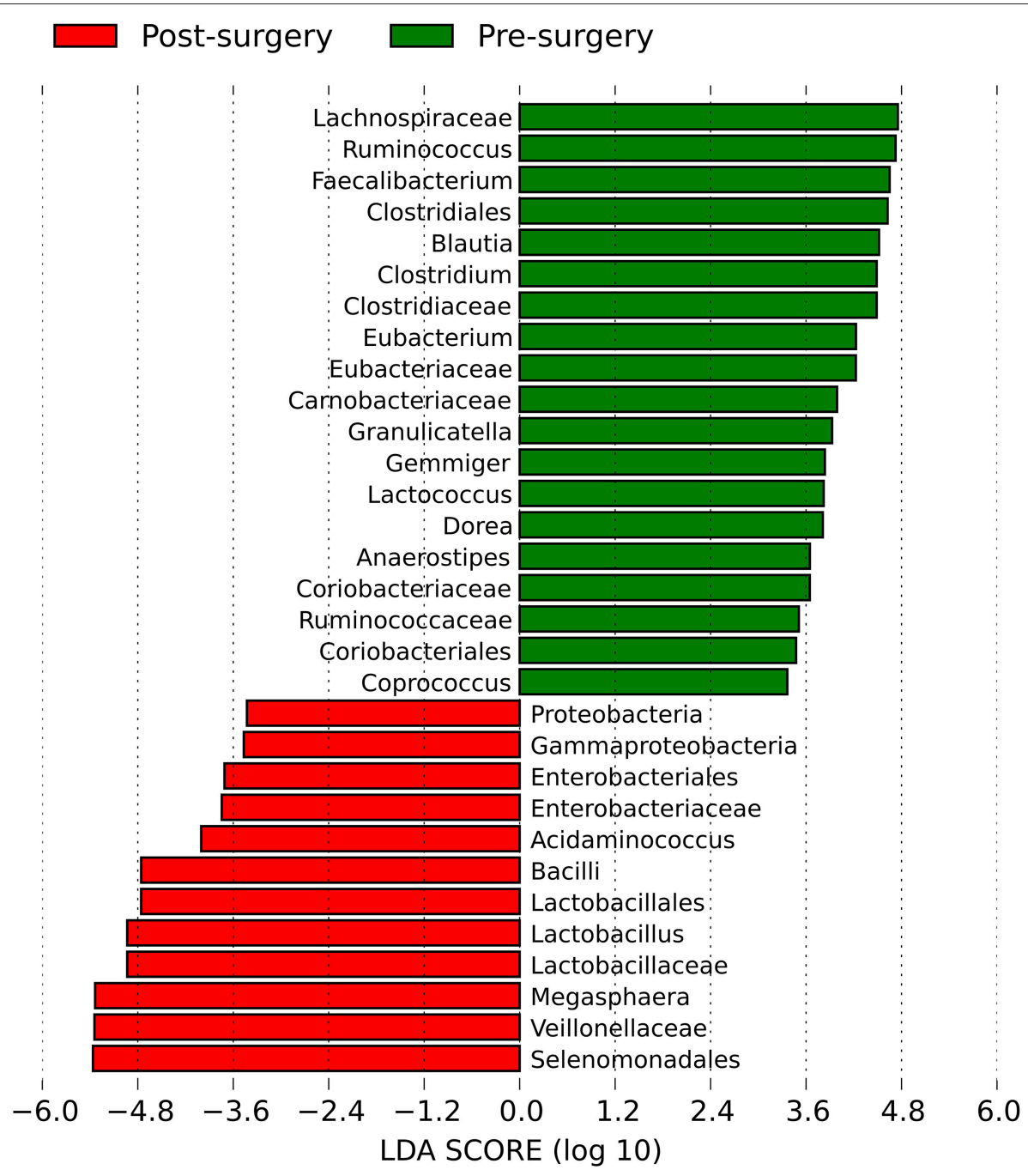

FIGURE 4 | Histogram of the linear discriminant analysis (LDA) scores for differentially abundant bacterial clades in fecal samples between obese individuals before and after bilio-intestinal bypass. Negative (red bars) LDA scores represent bacterial groups over-abundant in post-surgery samples while positive (green bars) represent bacterial groups overrepresented in pre-surgery samples.

$0.002)$, Gemmiger and serum glucose $(B=0.631, P=0.053)$, and total cholesterol and Clostridium $(B=0.696, P=0.141)$. There was a negative relationship between blood glucose and the relative abundance of Lactobacillus $(B=-0.551, P=0.141)$. To evaluate if such associations were influenced by variations in dietary calories, bivariate linear mixed models were fitted including caloric intake as a fixed effect. Only models in which the collinearity among predictors was not significant were run. Among these, associations between glucose and Gemmiger ( $B$ $=0.469, P=0.0006)$, glucose and Lactobacillus $(B=-0.385$, $P=0.0065)$, and triglycerides and Faecalibacterium $(B=$ $0.592, P=0.0002)$ remained significant even when controlling for caloric intake. In the first two aforementioned models, caloric intake displayed a significant positive association with glucose $(B=0.360$ and 0.347 , respectively, with associated $P=$ 0.0021 and 0.0079 , respectively), thus showing a simultaneous independent effect on blood glucose concentration; while, there was no significant association in the last model $(B=0.035$, $P=0.7066)$.

\section{Quantification of Functional Bacterial Groups}

Real-time PCR analysis was performed to detect bacterial functional groups whose metabolic activities play a key role in human colon metabolism. The qRT-PCR of the butyryl-CoA:acetate CoA-transferase (BCoAT) gene, involved in the microbial production of butyrate, indicated that the average abundance of the BcoAT gene was significantly reduced by about $2 \log$ in post-surgery subjects compared with pre-surgery (7.68 \pm 0.69 vs. $6.05 \pm 1.15 \log _{10}$ gene copies $g^{-1}, P=0.0048$ ) (Figure 5). Mean acetogenic bacteria levels were significantly lower in postsurgery subjects than in pre-surgery individuals (7.68 \pm 0.85 vs. $9.21 \pm 0.73 \log _{10}$ gene copies $\left.g^{-1}, P=0.0019\right)$ (Figure 5). Similarly, there was a significant post-surgery reduction in the 
mean number of methanogens $\left(4.93 \pm 0.70 \log _{10}\right.$ gene copies $\left.\mathrm{g}^{-1}\right)$ compared with baseline $\left(5.95 \pm 1.01 \log _{10}\right.$ gene copies $\mathrm{g}^{-1}$, $P=0.001)$. In contrast, quantitative PCR of the dissimilatory (bi)sulfite reductase gene revealed that mean levels of sulfatereducing bacteria were similar in the two groups (Figure 5).

\section{Bacterial Metabolism Products in Fecal Samples}

The $\mathrm{pH}$ of fecal samples was significantly lower in post-surgery subjects compared with pre-surgery $(5.72 \pm 0.70$ vs. $6.74 \pm$ $0.74, P=0.008)$. No statistically significant difference was found between the mean levels of urea in fecal samples from subjects pre-surgery compared with post-surgery (Figure 6). Both Llactate and D-lactate increased in post-surgery subjects; although, such differences did not reach statistical significance. Relative to pre-surgery samples, there were significantly lower levels of acetate $(42.63 \pm 16.34$ vs. $62.40 \pm 19.39 \%$ of total short chain fatty acids (SCFA), $P=0.0117$ ) and propionate (11.94 \pm 8.750 vs. $23.42 \pm 10.95 \%$ of total SCFA, $P=0.0049)$ in the post-surgery samples. On the contrary, the fecal samples from post-surgery individuals had significantly higher levels of valerate $(8.956 \pm 8.390$ vs. $2.258 \pm 1.660 \%$ of total SCFA, $P$ $=0.0162)$ and hexanoate $(4.117 \pm 4.364$ vs. $0.4600 \pm 0.7202 \%$ of total SCFA, $P=0.0039$ ) compared with pre-surgery samples (Figure 6). The fecal content of butyrate did not significantly vary in post-surgery patients compared with pre-surgery. When we tested for associations between the relative abundance of bacterial genera and fecal metabolite parameters, we observed significant positive relationships between hexanoate percentage levels and Megasphaera $(B=0.899, P=0.0008)$ and valerate percentage levels and Megasphaera $(B=0.773, P=0.04)$.

\section{DISCUSSION}

In our study, obese patients had high mean levels of Firmicutes and low levels of Bacteroidetes in their fecal samples, when evaluated by Illumina $16 \mathrm{~S}$ rDNA sequencing. Several authors have pointed out that human obesity is associated with high gut concentrations of Firmicutes and low concentrations of Bacteroidetes compared with lean individuals (Ley et al., 2006; Turnbaugh et al., 2009). This finding was not supported by other studies (Duncan et al., 2008; Schwiertz et al., 2010) and a recent analysis of data retrieved from the Human Microbiome Project (HMP) and MetaHIT survey showed no association between obesity and the Bacteroidetes-to-Firmicutes ratio (Finucane et al., 2014). Notably, there are reports of reduced Firmicutes and increased Bacteroidetes in obese subjects after weight loss induced by either gastric bypass (Zhang et al., 2009; Kong et al., 2013) or dietary restriction (Ley et al., 2006; Duncan et al., 2008). In this study, we did not observe either a decrease in Firmicutes abundance or an increase in Bacteroidetes in BIB-treated patients after surgery, i.e., the Bacteroidetes and Firmicutes bacterial populations shifting to proportions more like those usually found in lean individuals. Interestingly, the amounts of fecal Bacteroidetes detected in our study population were very much lower than those previously estimated in human obese individuals. The source of this discrepancy is unknown, but potential factors influencing the observed pattern include obesity grade, comorbid conditions, energy intake, age, population size, geographical location.

At lower taxonomic rank, we found several bacterial features that most likely explain the differences between pre- and post-surgery fecal microbiota. The bacterial genera within the Firmicutes phylum that were abundant in obese individuals
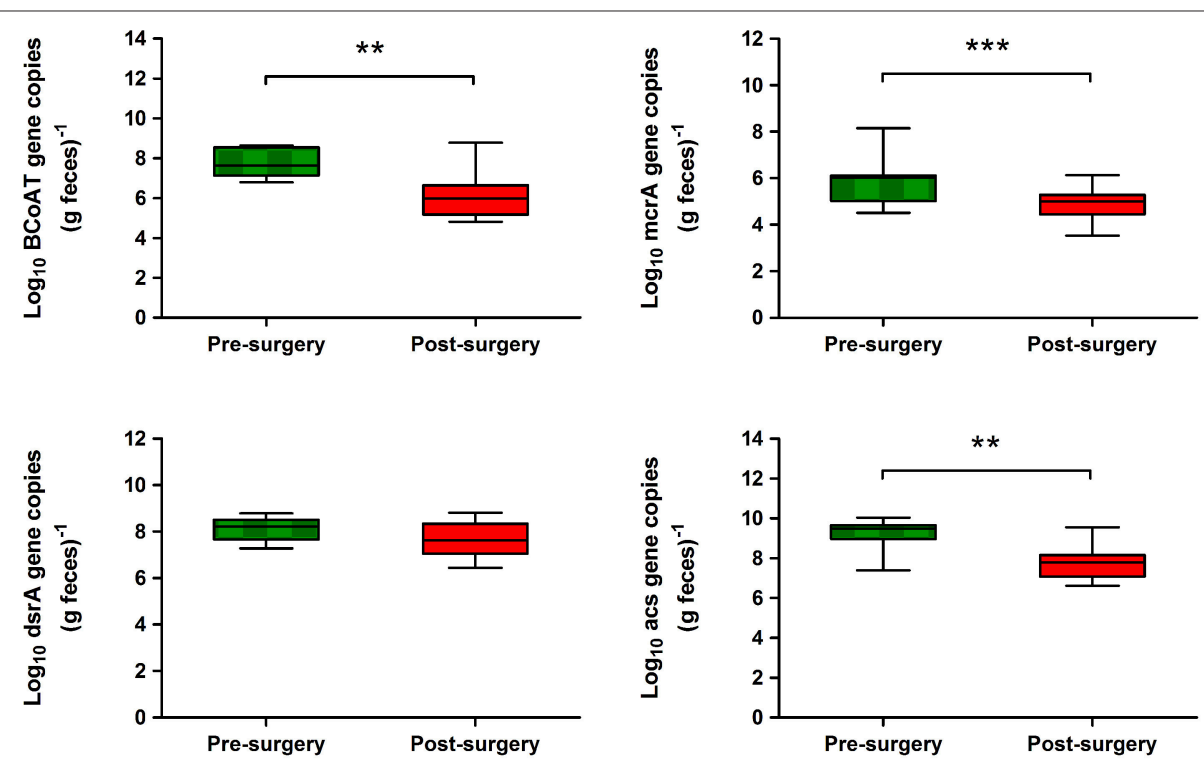

FIGURE 5 | Abundance of metabolic functional genes in the fecal microbiota of obese individuals before and after bariatric surgery as determined by qRT-PCR. Genes targeted included BcoAT for butyrate producers, acs for acetogens, $m c r A$ for methanogens, and dsrA for sulfate reducers. Values are log means \pm SD. Paired Student's $t$-test was performed on log-transformed data. Asterisks indicate statistical significance $\left({ }^{\star \star} P<0.01 ;{ }^{\star \star \star} P<0.001\right)$. 


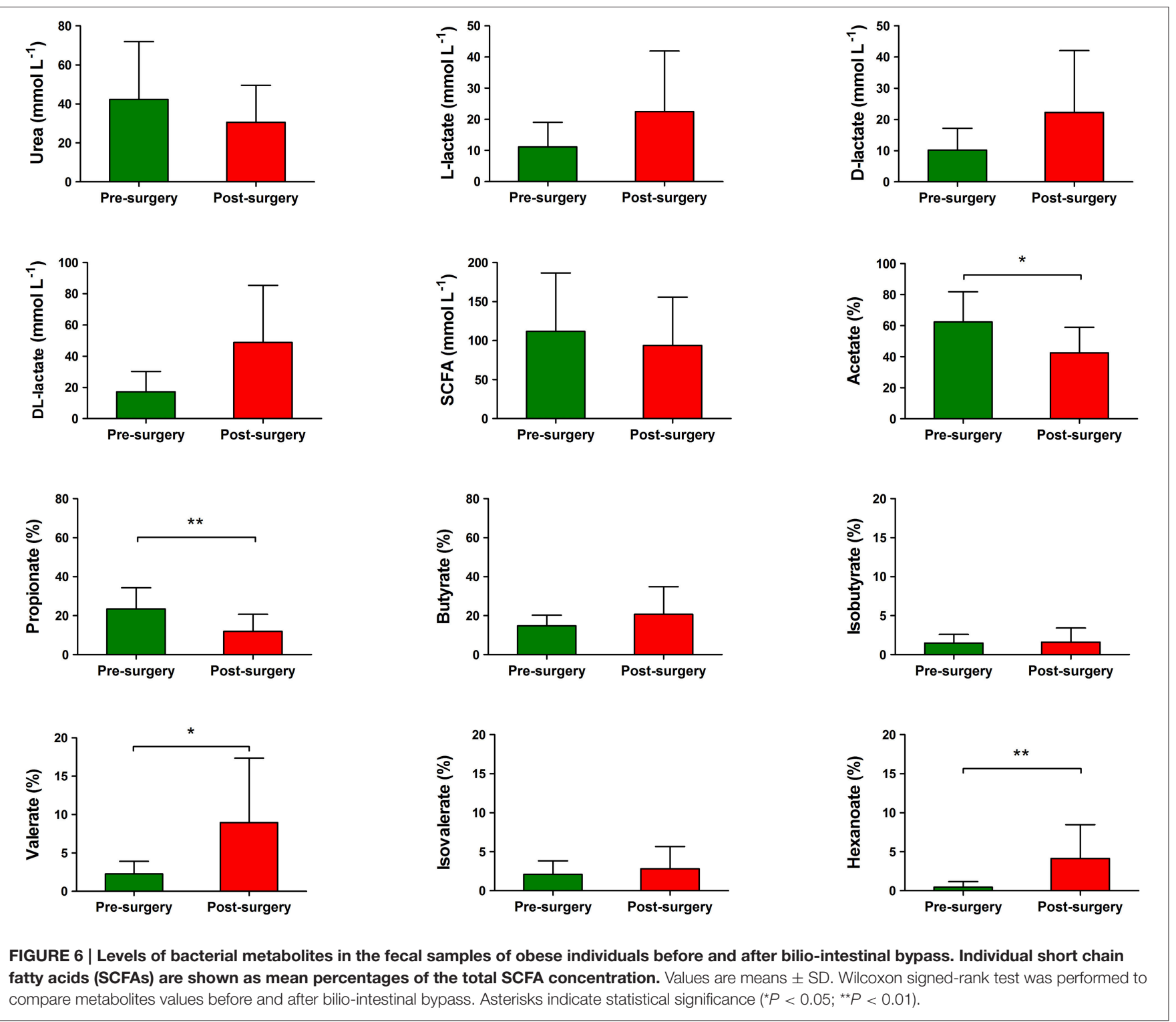

included mostly members of the order Clostridiales. Several authors have reported enrichment of Clostridium cluster XIVa (Verdam et al., 2013), Roseburia intestinalis (Tims et al., 2013; Verdam et al., 2013), and Eubacterium rectale (Ferrer et al., 2013; Tims et al., 2013) in the gut of obese subjects. In addition, pre-surgery obese individuals had increased Coriobacteriaceae. Members of the family Coriobacteriaceae represent dominant gut bacteria that are not well studied; although, they have important metabolic functions such as the conversion of bile salts and steroids that may influence host lipid metabolism. In support of this, studies in rodents determined that the abundance of this bacterial group significantly correlated with triglyceride levels or non-HDL cholesterol levels (Martínez et al., 2009; Claus et al., 2011). In post-surgery patients, the LefSE method showed a significant increase in Proteobacteria (Gammaproteobacteria), specifically Enterobacteriaceae, compared with pre-surgery. This result is in agreement with previous studies on the effects of
Roux-en-Y gastric bypass on gut microbiota that showed a substantial shift toward higher concentrations of Proteobacteria after surgery in both humans and rats (Zhang et al., 2009; Li et al., 2011; Graessler et al., 2013; Kong et al., 2013). In addition, the fecal microbiota of post-surgery patients was significantly enriched in bacterial taxa belonging to the order Lactobacillales and Selenomonadales, in particular the genera Lactobacillus, Acidaminococcus, and Megasphaera. The Lactobacillus spp. produce lactic acid and derive energy from the fermentation of lactose, glucose, and other sugars; while, Acidaminococcus spp. are among the predominant amino acid-fermenting microbiota along the digestive tract of humans and animals. Glucose and amino acid absorption mainly take place in the small intestine, so it is tempting to speculate that small intestinal resection, by increasing the amount of simple sugars and amino acids reaching the large intestine, may trigger colon bacteria that can use malabsorbed nutrients as an energy source. This mechanism has been 
described in human short bowel syndrome, where fermentation of non-digested carbohydrates in the large bowel can lead to a decrease in luminal $\mathrm{pH}$ as a result of the overgrowth of D-lactate producing bacteria, such as Lactobacillus spp. (Buchman et al., 2003; Zhang et al., 2003). Consistent with this, we observed a significant decrease of $\mathrm{pH}$ in fecal samples from post-surgery individuals, together with an increase in lactic acid, although the latter did not reach statistical significance. It seems also likely that an increased activity of lactate-producing bacteria in the distal gut may be linked to the augmented numbers of Megasphaera after surgery. Megasphaera elsdenii, the most studied of the Megasphaera spp., is known to actively ferment lactate in the rumen, thereby reducing acidosis in ruminants (Marounek et al., 1989). It has been suggested that Megasphaera spp. isolated from the human gut may serve a similar function (Shetty et al., 2013).

To gain further insight into the microbial community shifts associated with BIB, we evaluated the abundances of functional bacterial groups. We detected a significant decrease in the $B C o A T$ gene post-surgery. This result is not unexpected, since production of butyrate through BCoAT appears to be a common underlying feature of bacteria within the class Clostridia (Louis et al., 2010). Among hydrogenotrophic gut bacteria, a significant decrease of acetogenic bacteria was found after BIB. These results are in line with data from Illumina sequencing of $16 \mathrm{~S}$ rRNA gene amplicons, in that several bacterial groups that were significantly reduced in stool from post-surgery patients, including the genera Ruminococcus, Clostridium, and Blautia, are known acetate producers (Chassard and Bernalier-Donadille, 2006). Analogously, we found a reduction of methanogenic Archaea after bariatric surgery using quantitative PCR with $m c r A$ primers. These findings confirm results from Furet et al. (2010) and Zhang et al. (2009). Our results from acetogenic and methanogenic populations seem to further support the hypothesis of Zhang et al. (2009) that, in obese individuals, methanogens, and likely acetogens, accelerate the fermentation of indigestible polysaccharides by lowering the partial pressure of hydrogen. Thus, the energy harvesting from the diet is increased. Notably, we did not find any significant changes in the levels of sulfate-reducing bacteria in post-surgery patients compared with pre-surgery. One explanation for this is that the metabolism of sulfate-reducing bacteria is much more versatile than that of acetogens and methanogens since they can oxidize a variety of organic compounds such as lactate (Marquet et al., 2009) and can persist under different environmental conditions (Muyzer and Stams, 2008).

The observed shifts in bacterial populations between obese subjects before and after BIB led us to investigate potential changes in the gut environment conditions and fermentation parameters. The post-surgical decrease of $\mathrm{pH}$ points toward an altered intestinal environment that could reasonably influence gut microbial ecology, as indicated previously (Walker et al., 2005). Studies in animal models have shown that the obese microbiota differs from the lean microbiota and may produce more SCFAs and, hence, extract more energy from a given diet than the lean microbiota (Turnbaugh et al., 2006). Recent studies have also shown that fecal SCFA concentrations were significantly higher in obese subjects compared with lean participants (Schwiertz et al., 2010). However, in our study, we did not detect any significant variations in the mean levels of total SCFAs and butyrate in obese individuals before and after bariatric surgery. Although, it must be stressed that a real-time PCR assay targeting the BcoAT gene indicated a decrease of butyrate producers in the gut of surgical patients. In a recent study conducted in swine, several isolated strains, including $M$. elsdenii, did not have BcoAT genes detectable by degenerate PCR assay; although, they all had BcoAT enzyme activity. Authors suggested that the degenerate primers for BcoAT genes could underestimate the abundance of butyrate-producing bacteria and should be broadened to capture more butyrate producers in the gut (Levine et al., 2013). In the study by Liou et al. (2013), the RYGB mice had decreased total SCFAs compared with sham mice, with reductions in acetate levels and a relative increase in propionate levels. In our obese patients, the ratios of individual SCFAs are altered, with decreased acetate and propionate as well as increased valerate and hexanoate fecal concentrations. Such compounds are related to gut bacterial metabolism; thus, it is likely that variations in the SCFA profile of post-surgery patients reflect the specific composition of the gut microbial community. When we attempted to find linear relationships between bacterial groups and metabolites, we found that hexanoate and valerate levels were significantly associated with the relative proportion of Megasphaera spp. This is not surprising, since the rumen bacterium $M$. elsdenii reportedly produces valerate and caproate from redox-neutral substrates (sugars or lactate) (Marounek et al., 1989). It is can be hypothesized that the final concentrations of butyrate, acetate, and propionate in feces are the result of not only shifts in the direct production by microbial populations, but also of the interspecies cross-feeding interactions occurring in the distal gut. Further studies are necessary to elucidate the potential contributions of the specific microbial-derived metabolite changes on host colonic function and energy balance after BIB. In this regard, butyrate is the major energy source among SCFAs for colonocytes; while, acetate and propionate are absorbed into portal circulation. Propionate is largely taken up by the liver and consumed for gluconeogenesis; however, acetate is metabolized by peripheral tissues for de novo lipogenesis (Wong et al., 2006). Jørgensen et al. (1997) suggested that valerate and caproate are excellent substrates for oxidation in rat colonocytes, similar to butyrate. In addition to being used as an energy source for the host, the signaling properties of SCFAs could indicate a putative contribution to the changes associated with patients before and after the intervention. The SCFAs are ligands for the G protein-coupled receptors Gpr41 and Gpr43. Acetate exhibits higher potency at the human GPR43, whereas valerate and caproate have higher potency at human GPR41 (Brown et al., 2003; Le Poul et al., 2003). Notably, a growing body of evidence links GPR43 to body weight and metabolic disorders (Hong et al., 2005; Ge et al., 2008; Bjursell et al., 2011). Data in several animal studies have highlighted a role of GPR41 in regulating host energy balance, in particular by acting on sympathetic activity and intestinal gluconeogenesis (Samuel et al., 2008; Kimura et al., 2011; De Vadder et al., 2014).

Despite the small size of the study cohort, we succeeded in highlighting a few significant associations between alterations 
in metabolic indices and changes in the composition of the gut microbiota. In particular, Lactobacillus, which was upregulated after surgery, was negatively correlated with blood glucose. When looking at probiotic applications in humans and animal models, Lactobacillus spp. are effective in the regulation of glucose metabolism (Panwar et al., 2013). We found positive associations involving bacterial genera that decreased after BIB, such as Clostridium and blood insulin and total cholesterol, Faecalibacterium and triglycerides, and Gemmiger and blood glucose. Since dietary intake was significantly reduced after surgery, we verified such associations by controlling for the potential confounding effect of diet. After the statistical adjustment for caloric intake, these associations remained significant, indicating that the highlighted relationships between these bacterial populations (with the exception of Clostridium, which showed significant collinearity with caloric intake) and clinical parameters were independent of caloric intake. These results are in agreement with those of Kong et al. (2013) that found the populations of gut microbiota can be modulated via bariatric surgery independently of the variation in caloric consumption (Kong et al., 2013).

The present study has some limitations that have to be acknowledged. Obese patients experienced a marked decrease of body weight subsequent to the BIB as well as a significant reduction in caloric intake. The present sample size of 11 may be insufficient for highlighting every significant correlation pattern between gut microbiota and clinical parameters, and the observed correlations may be partly associated with other confounding variables. Regardless of the relevance of BIB for clinical practice, our study contributed to the identification of gut microbial populations and metabolite profiles that could represent putative markers of obesity, as well as markers of weight loss and metabolic improvement following bariatric surgery. These findings warrant further investigation to evaluate the exact impact of surgically-induced changes in human gut microbiota on host physiology and health. A deeper understanding of host-microbial crosstalk might help in the development of new alternative strategies as well as approaches to supplement

\section{REFERENCES}

Bäckhed, F., Ding, H., Wang, T., Hooper, L. V., Koh, G. Y., Nagy, A., et al. (2004). The gut microbiota as an environmental factor that regulates fat storage. Proc. Natl. Acad. Sci. U.S.A. 101, 15718-15723. doi: 10.1073/pnas.04070 76101

Bjursell, M., Admyre, T., Göransson, M., Marley, A. E., Smith, D. M., Oscarsson, J., et al. (2011). Improved glucose control and reduced body fat mass in free fatty acid receptor 2-deficient mice fed a high-fat diet. Am. J. Physiol. Endocrinol. Metabol. 300, E211-E220. doi: 10.1152/ajpendo.00229.2010

Brown, A. J., Goldsworthy, S. M., Barnes, A. A., Eilert, M. M., Tcheang, L., Daniels, D., et al. (2003). The orphan G protein-coupled receptors GPR41 and GPR43 are activated by propionate and other short chain carboxylic acids. J. Biol. Chem. 278, 11312-11319. doi: 10.1074/jbc.M211609200

Buchman, A. L., Scolapio, J., and Fryer, J. (2003). AGA technical review on short bowel syndrome and intestinal transplantation. Gastroenterology 124, 1111-1134. doi: 10.1016/S0016-5085(03)70064-X

Chassard, C., and Bernalier-Donadille, A. (2006). H2 and acetate transfers during xylan fermentation between a butyrate-producing xylanolytic species and bariatric surgery in the treatment of obesity and related comorbidities.

\section{AUTHOR CONTRIBUTIONS}

VP, AM, EV, ST, LD, AF, CL, and MD make substantial contributions to acquisition, analysis and interpretation of data. $\mathrm{VP}, \mathrm{AF}$, and AM participate in writing the article. MC, LM, and CL participate in critically revising the article.

\section{ACKNOWLEDGMENTS}

We are grateful to Dr. Sotirios Vasileiadis at University of South Australia for invaluable help on processing Illumina data.

\section{SUPPLEMENTARY MATERIAL}

The Supplementary Material for this article can be found online at: http://journal.frontiersin.org/article/10.3389/fmicb. 2016.00200

Supplementary Figure 1 | Normalized rarefaction curves displaying the average number of OTUs (at $97 \%$ similarity) discovered by random sampling within each fecal sample from obese subjects before (green line) and after (red line) bilio-intestinal bypass.

Supplementary Figure 2 | Hierarchical clustering (A) and a barplot of relative abundances (B) of bacterial phyla found within the fecal communities of obese patients. The dendrograms were calculated using Spearman's rank correlation and Ward-linkage clustering.

Supplementary Figure 3 | Cladogram indicating the phylogenetic distribution of differentially abundant bacterial lineages in fecal samples between obese individuals before and after bilio-intestinal bypass, determined by linear discriminant analysis (LDA). Red dots represent bacterial groups over-abundant in post-surgery samples, while green dots represent bacterial groups overrepresented in pre-surgery samples.

Supplementary Figure 4 | Biplot of the first two principal components from the principal component analysis (PCA) of bacterial genera relative abundances and bio-clinical markers. The length of the vectors represents the PCA loadings of the variables on the first two principal components. Dots represent individual subjects before (solid circles) and after (open circles) bilio-intestinal bypass. hydrogenotrophic microorganisms from the human gut. FEMS Microbiol. Lett. 254, 116-122. doi: 10.1111/j.1574-6968.2005.00016.x

Claesson, M. J., O'Sullivan, O., Wang, Q., Nikkilä, J., Marchesi, J. R., Smidt, H., et al. (2009). Comparative analysis of pyrosequencing and a phylogenetic microarray for exploring microbial community structures in the human distal intestine. PLoS ONE 4:e6669. doi: 10.1371/journal.pone.0006669

Claus, S. P., Ellero, S. L., Berger, B., Krause, L., Bruttin, A., Molina, J., et al. (2011). Colonization-induced host-gut microbial metabolic interaction. MBio 2, e00271-e00210. doi: 10.1128/mBio.00271-10

Denman, S. E., Tomkins, N., and McSweeney, C. S. (2007). Quantitation and diversity analysis of ruminal methanogenic populations in response to the antimethanogenic compound bromochloromethane. FEMS Microbiol. Ecol. 62, 313-322. doi: 10.1111/j.1574-6941.2007.00394.x

De Vadder, F., Kovatcheva-Datchary, P., Goncalves, D., Vinera, J., Zitoun, C., Duchampt, A., et al. (2014). Microbiota-generated metabolites promote metabolic benefits via gut-brain neural circuits. Cell 156, 84-96. doi: 10.1016/j.cell.2013.12.016

Duncan, S. H., Lobley, G. E., Holtrop, G., Ince, J., Johnstone, A. M., and Louis, P. (2008). Human colonic microbiota associated with diet, obesity 
and weight loss. Int. J. Obes. (Lond). 32, 1720-1724. doi: 10.1038/ijo.200 8.155

Eriksson, F. (1981). Biliointestinal bypass. Int. J. Obes. 5, 437-447.

Ferrer, M., Ruiz, A., Lanza, F., Haange, S. B., Oberbach, A., Till, H., et al. (2013). Microbiota from the distal guts of lean and obese adolescents exhibit partial functional redundancy besides clear differences in community structure. Environ. Microbiol. 15, 211-226. doi: 10.1111/j.1462-2920.2012.02845.x

Finucane, M. M., Sharpton, T. J., Laurent, T. J., and Pollard, K. S. (2014). A taxonomic signature of obesity in the microbiome? Getting to the guts of the matter. PLoS ONE 9:e84689. doi: 10.1371/journal.pone.0084689

Furet, J. P., Kong, L. C., Tap, J., Poitou, C., Basdevant, A., Bouillot, J. L., et al. (2010). Differential adaptation of human gut microbiota to bariatric surgeryinduced weight loss, links with metabolic and low-grade inflammation markers. Diabetes 59, 3049-3057. doi: 10.2337/db10-0253

Gagen, E. J., Denman, S. E., Padmanabha, J., Zadbuke, S., Al Jassim, R., Morrison, M., et al. (2010). Functional gene analysis suggests different acetogen populations in the bovine rumen and tammar wallaby forestomach. Appl. Environ. Microbiol. 76, 7785-7795. doi: 10.1128/AEM.01679-10

Ge, H. F., Li, X. F., Weiszmann, J., Wang, P., Baribault, H., Chen, J. L., et al. (2008). Activation of $\mathrm{G}$ protein-coupled receptor 43 in adipocytes leads to inhibition of lipolysis and suppression of plasma free fatty acids. Endocrinology 149, 4519-4526. doi: 10.1210/en.2008-0059

Graessler, J., Qin, Y., Zhong, H., Zhang, J., Licinio, J., Wong, M. L., et al. (2013). Metagenomic sequencing of the human gut microbiome before and after bariatric surgery in obese patients with type 2 diabetes, correlation with inflammatory and metabolic parameters. Pharmacogenomics J. 13, 514-522. doi: $10.1038 /$ tpj.2012.43

Hong, Y. H., Nishimura, Y., Hishikawa, D., Tsuzuki, H., Miyahara, H., Gotoh, C., et al. (2005). Acetate and propionate short chain fatty acids stimulate adipogenesis via GPCR43. Endocrinology 146, 5092-5099. doi: 10.1210/en.2005-0545

Jørgensen, J. R., Clausen, M. R., and Mortensen, P. B. (1997). Oxidation of short and medium chain C2-C8 fatty acids in Sprague-Dawley rat colonocytes. Gut 40, 400-405. doi: 10.1136/gut.40.3.400

Kimura, I., Inoue, D., Maeda, T., Hara, T., Ichimura, A., Miyauchi, S., et al. (2011). Short-chain fatty acids and ketones directly regulate sympathetic nervous system via G protein-coupled receptor 41 (GPR41). Proc. Natl. Acad. Sci. U.S.A. 108, 8030-8035. doi: 10.1073/pnas.1016088108

Klindworth, A., Pruesse, E., Schweer, T., Peplies, J., Quast, C., Horn, M., et al. (2013). Evaluation of general 16S ribosomal RNA gene PCR primers for classical and next-generation sequencing-based diversity studies. Nucleic Acids Res. 41:e1. doi: 10.1093/nar/gks808

Kondo, R., Nedwell, D. B., Purdy, K. J., and Silva, S. D. (2004). Detection and enumeration of sulphate-reducing bacteria in estuarine sediments by competitive PCR. Geomicrobiol. J. 21, 145-157. doi: 10.1080/01490450490275307

Kong, L. C., Tap, J., Aron-Wisnewsky, J., Pelloux, V., Basdevant, A., Bouillot, J. L., et al. (2013). Gut microbiota after gastric bypass in human obesity, increased richness and associations of bacterial genera with adipose tissue genes. Am. J. Clin. Nutr. 98, 16-24. doi: 10.3945/ajcn.113.058743

Le Poul, E., Loison, C., Struyf, S., Springael, J. Y., Lannoy, V., Decobecq, M. E., et al. (2003). Functional characterization of human receptors for short chain fatty acids and their role in polymorphonuclear cell activation. J. Biol. Chem. 278, 25481-25489. doi: 10.1074/jbc.M301403200

Levine, U. Y., Looft, T., Allen, H. K., and Stanton, T. B. (2013). Butyrate-producing bacteria, including mucin degraders, from the swine intestinal tract. Appl. Environ. Microbiol. 79, 3879-3881. doi: 10.1128/AEM.00589-13

Ley, R. E., Bäckhed, F., Turnbaugh, P., Lozupone, C. A., Knight, R. D., and Gordon, J. I. (2005). Obesity alters gut microbial ecology. Proc. Natl. Acad. Sci. U.S.A. 102, 11070-11075. doi: 10.1073/pnas.0504978102

Ley, R. E., Turnbaugh, P. J., Klein, S., and Gordon, J. I. (2006). Microbial ecology: human gut microbes associated with obesity. Nature 444, 1022-1023. doi: 10.1038/4441022a

Li, J. V., Ashrafian, H., Bueter, M., Kinross, J., Sands, C., Le Roux, C. W., et al. (2011). Metabolic surgery profoundly influences gut microbial-host metabolic cross-talk. Gut 60, 1214-1223. doi: 10.1136/gut.2010.234708

Liou, A., Paziuk, M., Luevano, J., Machineni, S., Turnbaugh, P., and Kaplan, L. (2013). Conserved shifts in the gut microbiota due to gastric bypass reduce host weight and adiposity. Sci. Transl. Med. 5:178ra41. doi: 10.1126/scitranslmed.3005687

Louis, P., and Flint, H. J. (2007). Development of a semiquantitative degenerate real-time PCR-based assay for estimation of numbers of butyryl-coenzyme A (CoA) CoA transferase genes in complex bacterial samples. Appl. Environ. Microbiol. 73, 2009-2012. doi: 10.1128/AEM.02561-06

Louis, P., Young, P., Holtrop, G., and Flint, H. J. (2010). Diversity of human colonic butyrate producing bacteria revealed by analysis of the butyryl-CoA:acetate CoA-transferase gene. Environ. Microbiol. 12, 304-314. doi: 10.1111/j.14622920.2009.02066.x

Marounek, M., Fliegrova, K., and Bartos, S. (1989). Metabolism and some characteristics of ruminal strains of Megasphaera elsdenii. Appl. Environ. Microbiol. 55, 1570-1573.

Marquet, P., Duncan, S. H., Chassard, C., Bernalier-Donadille, A., and Flint, H. J. (2009). Lactate has the potential to promote hydrogen sulphide formation in the human colon. FEMS Microbiol. Lett. 299, 128-134. doi: 10.1111/j.15746968.2009.01750.x

Martínez, I., Wallace, G., Zhang, C., Legge, R., Benson, A. K., Carr, T. P., et al. (2009). Diet-induced metabolic improvements in a hamster model of hypercholesterolemia are strongly linked to alterations of the gut microbiota. Appl. Environ. Microbiol. 75, 4175-4184. doi: 10.1128/AEM.00380-09

Musso, G., Gambino, R., Durazzo, M., Biroli, G., Carello, M., Fagà, E., et al. (2005). Adipokines in NASH: post prandial lipid metabolism as a link between adiponectin and liver disease. Hepatology 42, 1175-118383. doi: 10.1002/hep.20896

Muyzer, G., and Stams, A. J. M. (2008). The ecology and biotechnology of sulphatereducing bacteria. Nat. Rev. Microbiol. 6, 441-454. doi: 10.1038/nrmicro1892

Oksanen, J., Blanchet, F. G., Kindt, R., Legendre, P., Minchin, P. R., O’Hara, R. B., et al. (2014). Vegan, Community Ecology Package. R Package Version 2.2-0.

Panwar, H., Rashmi, H. M., Batish, V. K., and Grover, S. (2013). Probiotics as potential biotherapeutics in the management of type 2 diabetes - prospects and perspectives. Diabetes Metab. Res. Rev. 29, 103-112. doi: 10.1002/dmrr.2376

Pinheiro, J., Bates, D., DebRoy, S., Sarkar, D., and R Core Team (2013). nlme, Linear and Nonlinear Mixed Effects Models. R package version 3.1-107.

R Core Team (2014). R: A Language and Environment for Statistical Computing. Vienna: R Foundation for Statistical Computing.

Samuel, B. S., Shaito, A., Motoike, T., Rey, F. E., Bäckhed, F., Manchester, J. K., et al. (2008). Effects of the gut microbiota on host adiposity are modulated by the short-chain fatty-acid binding G protein-coupled receptor, Gpr41. Proc. Natl. Acad. Sci. U.S.A. 105, 16767-16772. doi: 10.1073/pnas.0808567105

Schwiertz, A., Taras, D., Schäfer, K., Beijer, S., Bos, N. A., Donus, C., et al. (2010). Microbiota and SCFA in lean and overweight healthy subjects. Obesity 18, 190-195. doi: 10.1038/oby.2009.167

Segata, N., Izard, J., Waldron, L., Gevers, D., Miropolsky, L., Garrett, W. S., et al. (2011). Metagenomic biomarker discovery and explanation. Genome Biol. 12:R60. doi: 10.1186/gb-2011-12-6-r60

Shetty, S. A., Marathe, N. P., Lanjekar, V., Ranade, D., and Shouche, Y. S. (2013). Comparative genome analysis of Megasphaera sp. reveals niche specialization and its potential role in the human gut. PLOS ONE 8:e79353. doi: 10.1371/journal.pone.0079353

Tims, S., Derom, C., Jonkers, D. M., Vlietinck, R., Saris, W. H., Kleerebezem, M., et al. (2013). Microbiota conservation and BMI signatures in adult monozygotic twins. ISME J. 7, 707-717. doi: 10.1038/ismej.2012.146

Turnbaugh, P. J., Bäckhed, F., Fulton, L., and Gordon, J. I. (2008). Diet-induced obesity is linked to marked but reversible alterations in the mouse distal gut microbiome. Cell Host Microbe 3, 213-223. doi: 10.1016/j.chom.2008.02.015

Turnbaugh, P. J., Hamady, M., Yatsunenko, T., Cantarel, B. L., Duncan, A., Ley, R. E., et al. (2009). A core gut microbiome in obese and lean twins. Nature 457, 480-484. doi: 10.1038/nature07540

Turnbaugh, P. J., Ley, R. E., Mahowald, M. A., Magrini, V., Mardis, E. R., and Gordon, J. I. (2006). An obesity-associated gut microbiome with increased capacity for energy harvest. Nature 444, 1027-1031. doi: 10.1038/nature05414

Verdam, F. J., Fuentes, S., de Jonge, C., Zoetendal, E. G., Erbil, R., Greve, J. W., et al. (2013). Human intestinal microbiota composition is associated with local and systemic inflammation in obesity. Obesity 21, E607-E615. doi: 10.1002/oby.20466

Walker, A. W., Duncan, S. H., Leitch, E. C. M., Child, M. W., and Flint, H. J. (2005). $\mathrm{pH}$ and peptide supply can radically alter bacterial populations and short-chain 
fatty acid ratios within microbial communities from the human colon. Appl. Environ. Microbiol. 71, 3692-3700. doi: 10.1128/AEM.71.7.3692-3700.2005

Wong, J. M., de Souza, R., Kendall, C. W., Emam, A., and Jenkins, D. J. (2006). Colonic health: fermentation and short chain fatty acids. J. Clin. Gastroenterol. 40, 235-243. doi: 10.1097/00004836-200603000-00015

Zhang, D. L., Jiang, Z. W., Jiang, J., Cao, B., and Li, J. S. (2003). D-lactic acidosis secondary to short bowel syndrome. Postgrad. Med. J. 79, 110-112. doi: 10.1136/pmj.79.928.110

Zhang, H., DiBaise, J. K., Zuccolo, A., Kudrna, D., Braidotti, M., Yu, Y., et al. (2009). Human gut microbiota in obesity and after gastric bypass. Proc. Natl. Acad. Sci. U.S.A. 106, 2365-2370. doi: 10.1073/pnas.0812600106
Conflict of Interest Statement: The authors declare that the research was conducted in the absence of any commercial or financial relationships that could be construed as a potential conflict of interest.

Copyright (๑) 2016 Patrone, Vajana, Minuti, Callegari, Federico, Loguercio, Dallio, Tolone, Docimo and Morelli. This is an open-access article distributed under the terms of the Creative Commons Attribution License (CC BY). The use, distribution or reproduction in other forums is permitted, provided the original author(s) or licensor are credited and that the original publication in this journal is cited, in accordance with accepted academic practice. No use, distribution or reproduction is permitted which does not comply with these terms. 